$19^{\text {th }}$ Australasian Fluid Mechanics Conference

Melbourne, Australia

8-11 December 2014

\title{
Spectral Content of Cloud Cavitation about a Sphere
}

\author{
K. L. de Graaf, P. A. Brandner and B. W. Pearce \\ Australian Maritime College, \\ University of Tasmania, Launceston, Tasmania 7250, Australia
}

\begin{abstract}
Cloud cavitation about a sphere is investigated in a variablepressure water tunnel using simultaneous high-speed photography and high-frequency surface pressure measurements. The $0.15 \mathrm{~m}$ diameter PVC sphere was located on the axial centreline of the $0.6 \mathrm{~m}$ square test section and tested at a constant Reynolds number of $1.5 \times 10^{6}$ and cavitation numbers ranging from 0.4 to 1.0. The flush-mounted high-frequency pressure sensor was located within the shedding zone of the cloud cavitation and orientated such that it was visible by the high-speed camera. Pressure measurements were recorded at $7 \mathrm{kHz}$. The high-speed photography was simultaneously obtained at $7 \mathrm{kHz}$ and a time-series of the pixel-intensity was extracted at the pressure sensor location. The spectral content of the cloud cavitation was obtained from frequency analysis of the time series from the surface pressure measurements and high-speed photography. The coherence of the two signals was measured using Pearson's Correlation Coefficient, for which the values ranged between 0.57 and 0.73 . Both data sets were investigated using wavelet analysis. Two peak frequencies are observable at most cavitation numbers tested that correspond to different shedding modes relating to cavity length.
\end{abstract}

\section{Introduction}

Cloud cavitation occurs when an attached cavity is periodically broken off due to the penetration of the re-entrant jet that forms in the cavity closure region. The cavity volumes broken off from the main cavity break down into a cloud of bubbbles as they are advected downstream [5]. This phenomenon is widely studied, e.g. [7, 8, 11, 12], however has generally been limited to hydrofoils. Brennen [4] studied cavity formation behind axisymmetric headforms (including spheres), investigating the surface appearance and nature of the separated interfacial boundary layer. Arakeri and Acosta [1] investigated cavitation inception and the role of boundary layer separation and turbulent reattachment. Huang $[9,10]$ also investigated cavitation inception in regions of boundary layer separation for six axisymmetric headforms. Ceccio and Brennen [6] studied the fluctu-

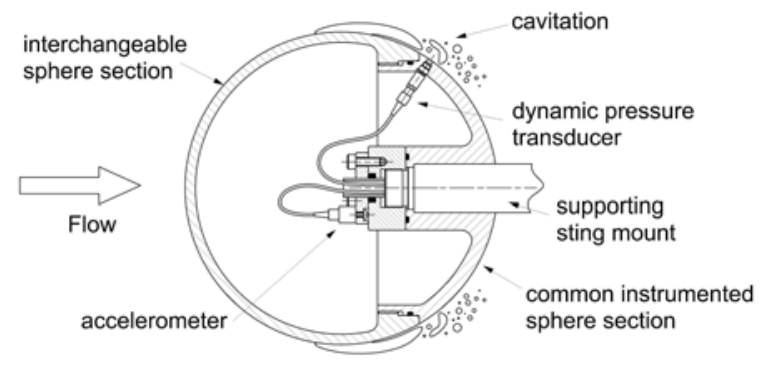

Figure 1. Sphere assembly.

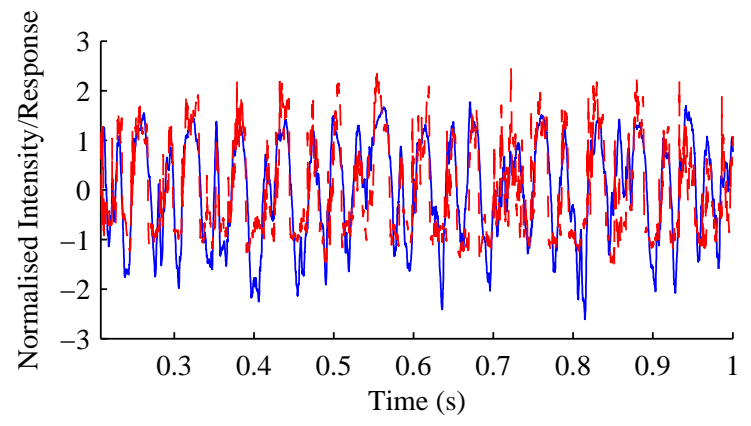

Figure 2. Simultaneous image pixel-intensity (blue, solid) and pressure transducer response (red, dashed) for $\sigma=0.8$. The image pixelintensity time series has been reverse polarised such that low image pixel-intensity corresponds with a high pressure signal from the transducer.

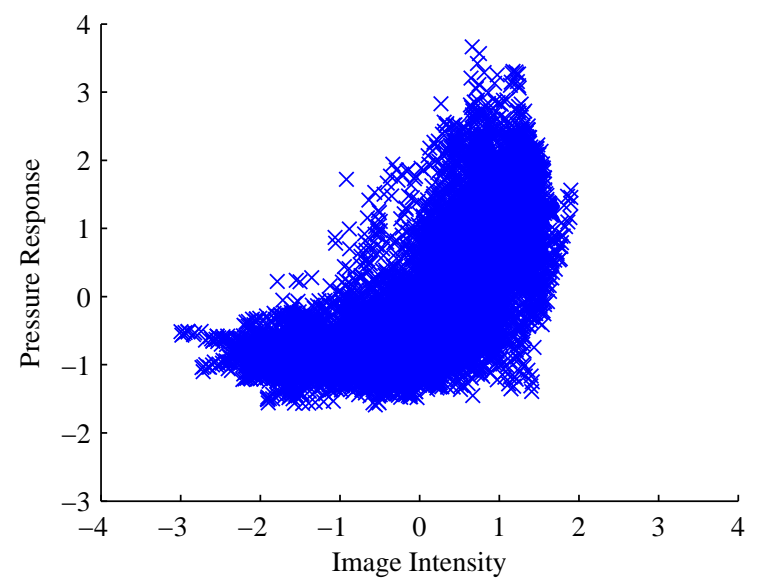

Figure 3. Scatter plot of normalised image pixel-intensity vs. pressure response for $\sigma=0.8$. The Pearson's Correlation Coefficient is 0.73.

ations of attached cavities on two axisymmetric bodies. Cloud cavitation about a sphere is not well reported on. The present work extends that of Brandner et al. [2] and Brandner et al. [3] and investigates the shedding frequencies of the cloud cavitation developed about a sphere at high Reynolds numbers using two diagnostic techniques: surface pressure measurements and high-speed photography. The results from the two techniques are compared and the merits of each technique identified. Both results provide information on the shedding frequencies, with the high-speed imaging providing high spatial resolution and details of the physics, and the pressure measurements providing high temporal resolution of the shedding events.

\section{Experimental Setup}

A $0.15 \mathrm{~m}$ diameter PolyVinylChlorate (PVC) sphere was mounted on the axial centreline of the test section of the Australian Maritime College (AMC) Cavitation Research Laboratory (CRL) variable-pressure water tunnel. The test section is 


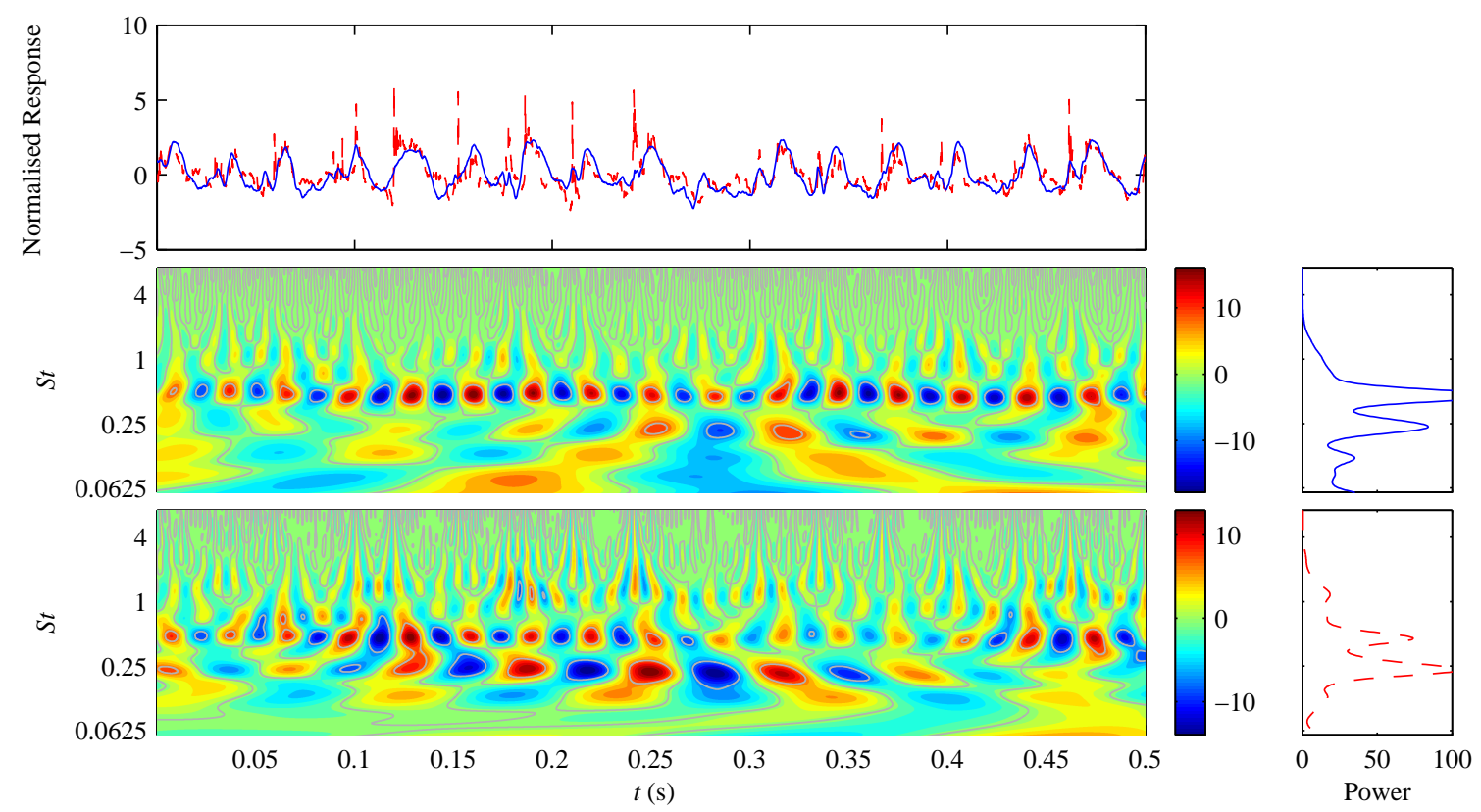

Figure 4. Time series of image pixel-intensity (blue, solid) and pressure (red, dashed) shown at top with Morlet-6 wavelet spectra and global wavelet spectra of image pixel-intensity (middle) and pressure (bottom) for $\sigma=0.8$. The same events can be seen from both measurement methods.

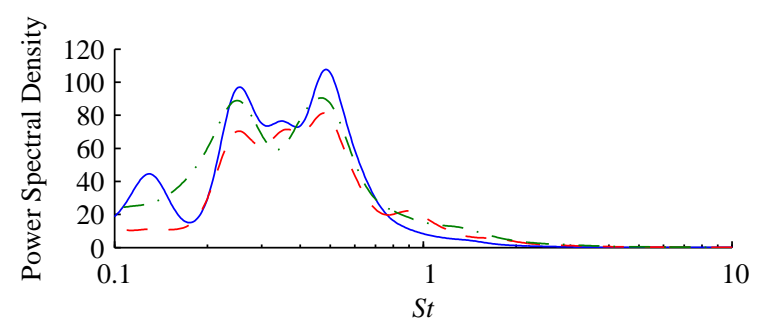

Figure 5. Wavelet spectra of short pressure time series (red, dashed), long pressure time series (green, dot-dash) and image pixel-intensity (blue, solid) for $\sigma=0.8$. The long pressure time series more accurately resolves the shedding spectra; however, the shorter series both identify the two shedding frequencies.

$0.6 \mathrm{~m}$ square and the tunnel pressure at the centreline can be varied from $4-400 \mathrm{kPa}$ (absolute) and the velocity from 2 $12 \mathrm{~m} / \mathrm{s}$. A flush-mounted PCB model $105 \mathrm{C} 02$ pressure sensor was mounted on the sphere in the shedding zone of the cavitation (at a 120 degree angle from the stagnation point, see figure 1). The sphere was orientated such that the sensor was visible in the high-speed photography. In this way the cavitation dynamics imaged are at the same location as the measured wall pressure. The measurements were made at $7 \mathrm{kHz}$ over $2 \mathrm{~s}$. In addition longer time series of pressure measurements were obtained at $1024 \mathrm{~Hz}$ for $200 \mathrm{~s}$. Data were recorded using a National Instruments PXIe-4497 card. High-speed photography was obtained using a LaVision HighSpeedStar8 camera, operated using DaVis 8.1 software and illuminated with a Dedolight DLH650. Low-speed still photographs were obtained using a Canon 50D $35 \mathrm{~mm}$ SLR camera and Canon EF 24-70 mm lens with triggered stroboscopic lighting (DRELLO 1018/LE4040)

\section{Results}

From the high-speed photography, time series of spatially averaged pixel intensities were extracted at the point of the pressure transducer. A window of $65 \times 65$ pixels was used to provide the required spatial resolution whilst minimising noise. The time series were shown to be relatively insensitive to window size.
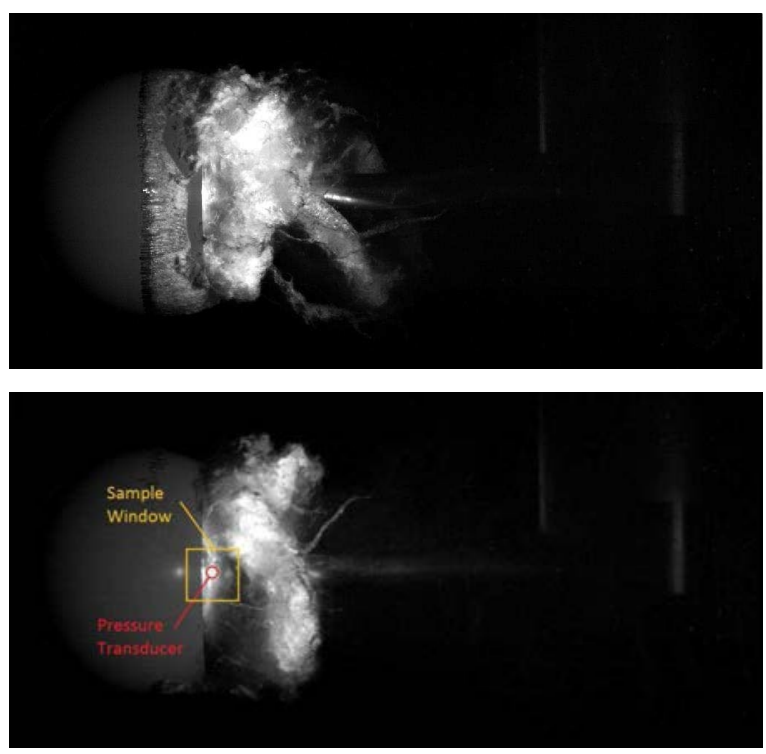

Figure 6. High-speed images of cavity shedding at $\sigma=0.8$. The top image shows a shorter duration shedding event at $t=0.87 \mathrm{~s}$. The bottom image shows a full cavity axisymmetric shedding event at $t=0.110 \mathrm{~s}$.

For these experiments a software error caused a delay between the trigger signal and the start of the camera recording. A crosscorrelation was performed on the two signals which was used to determine the delay and re-synchronise the signals. Figure 2 shows the simultaneous pressure measurement and image pixelintensity time series for $\sigma=0.8$. The cavitation number, $\sigma$, is given by: $\left(p-p_{v}\right) / \frac{1}{2} \rho U^{2}$, where $p$ is the tunnel pressure, $p_{v}$ is the water vapour pressure, $\rho$ is the water density and $U$ is the tunnel velocity. Both signals have had the mean subtracted and magnitude normalised by the standard deviation. The image pixel-intensity has been reverse polarised such that low image pixel-intensity corresponds with a high pressure signal from the transducer. 
The signals follow each other well. A typical scatter plot of the image pixel-intensity vs. the pressure response is shown in figure 3. The Pearson Correlation Coefficient $(P C C)$ is calculated by

$$
P C C=\frac{\sum_{i=1}^{n}\left[\left(x_{i}-\bar{x}\right)\left(y_{i}-\bar{y}\right)\right]}{\sqrt{\sum_{i=1}^{n}\left(x_{i}-\bar{x}\right)^{2} \sum_{i=1}^{n}\left(y_{i}-\bar{y}\right)^{2}}}
$$

where $n$ is the number of samples, $x$ and $y$ are the data points of the two signals and an overbar represents the mean. It varies between -1 and 1 where -1 is total negative correlation, 0 is no correlation and 1 is total positive correlation. The PCC of the image pixel-intensity and pressure response signals varies between 0.57 and 0.73 . On inspection of the signals, the image pixel-intensity shows the same frequency of the shedding events as the pressure response; however, the amplitudes are varied. The similarities of the signals for the shedding frequencies shows that the spectral content of the cloud cavitation can be measured using both high-speed imagery and surface pressure measurements. High-speed imagery allows visual inspection of the shedding events and comparison between event types and frequency content. Surface pressure measurements allow measurement of longer time series for improved spectral analysis, and give greater accuracy on the magnitude of the pressures generated.

The wavelet analysis method of Torrence and Compo [13] with a Morlet- 6 wavelet has been used to analyse the frequency content of the image pixel-intensity time series and both long and short pressure transducer measurements. Figure 4 shows the wavelet analysis of the image pixel-intensity time series and short pressure response at $\sigma=0.8$. The frequencies are represented as Strouhal numbers, given by $f D / U$, where $f$ is the frequency and $D$ is the sphere diameter. Two dominant frequencies are seen at $S t=0.25$ and and 0.47 . The comparison of the spectra from these time series with the spectra from the long pressure time series is shown in figure 5; all three spectra show the same peak frequencies, with reduction in noise at the lower frequencies evident in the spectra from the longer time series, as expected. From inspection of the high-speed movies, the lower frequency corresponds with full sheet cavity detachment or the axisymmetric mode identified in [3]. The higher frequency represents the intermediate or asymmetric shedding mode. Highspeed images are shown in figure 6 at $t=0.87 \mathrm{~s}$, where there is a shorter duration, asymmetric shedding event and $t=0.110 \mathrm{~s}$ where the full cavity is shed axisymmetrically.

Figures 7-8 show the wavelet analysis of image pixel-intensity at $\sigma=0.5$. Figure 9 shows low (top image) and high (bottom image) frequency shedding events. The PCC for the pressure response and image pixel-intensity is 0.63 .

As $\sigma$ reduces, a more permanent cavity is formed. At the higher $\sigma$ values, when the shedding is most energetic, the cavity leading edge is extensively eliminated for short periods, after which re-nucleation and cavity regrowth occurs. At lower $\sigma$ values the larger shedding events do not result in the extensive elimination of the cavity. The re-entrant jet that causes the cavity to shed does not fully eliminate the laminar cells that characterise the initial formation of the cavity [3] and the shed cavity remains connected via streamwise vortices.

\section{Conclusions}

Cloud cavitation about a PVC sphere was investigated using simultaneous high-speed photography and high-frequency surface pressure measurements. The coherence between the two measurements was found to be favourable, with $P C C$ values ranging between 0.57 and 0.73 . Wavelets were used to analyse the signals and identify cavity shedding events and frequencies. Both measurement techniques identified the same shedding events and frequencies, with the pressure measurement providing higher temporal resolution and the image pixelintensity provided details of the physics. Two dominant frequencies were found that represented larger and smaller shedding events. At high $\sigma$ the larger events are axisymmetric shedding of the full cavity. At lower $\sigma$ the cavity is generally not shed in its entirety, remaining connected via streamwise vortices.

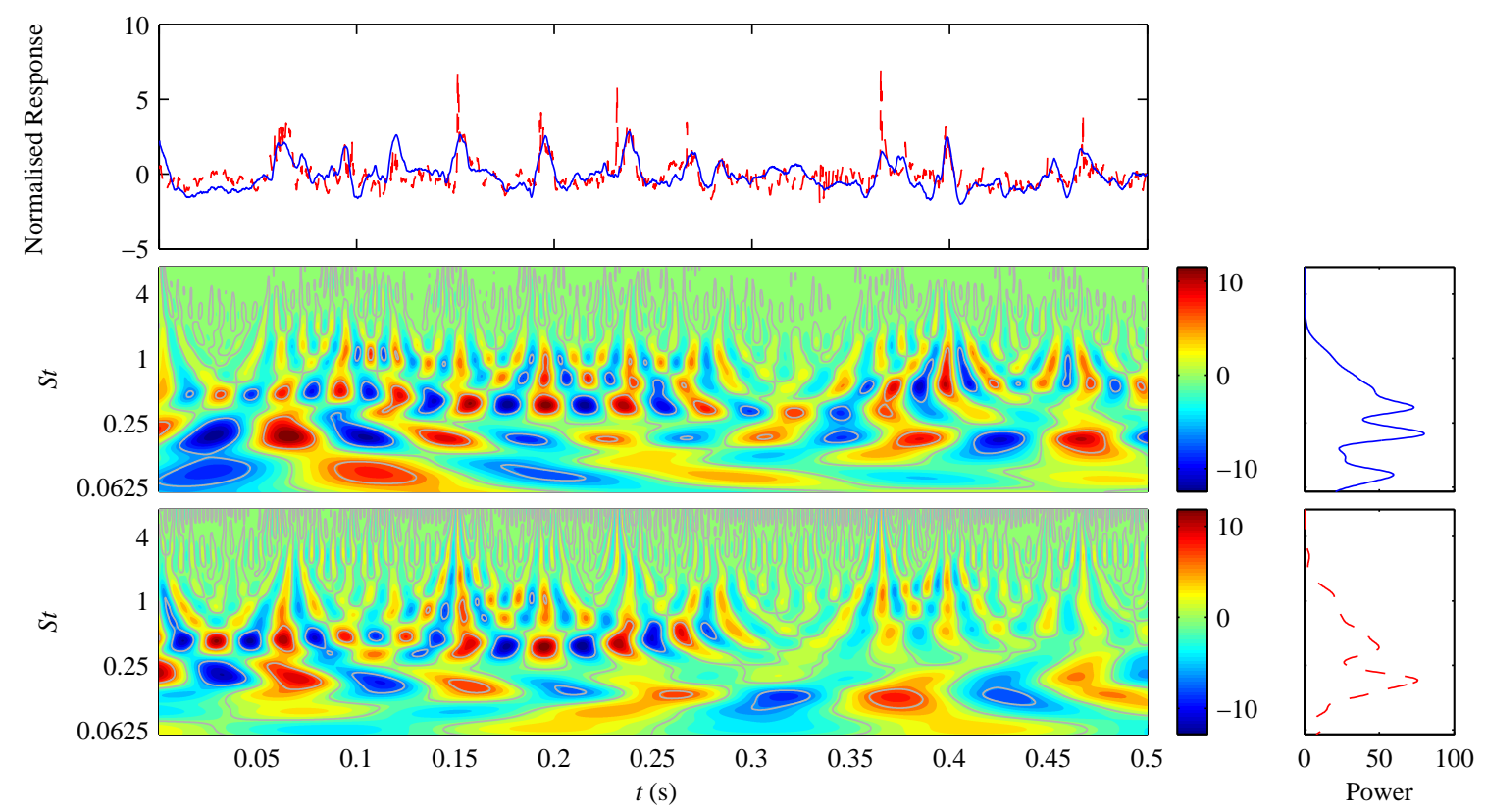

Figure 7. Time series of image pixel-intensity (blue, solid) and pressure (red, dashed) shown at top with Morlet-6 wavelet spectra and global wavelet spectra of image pixel-intensity (middle) and pressure (bottom) for $\sigma=0.5$. The same events can be seen from both measurement methods. 


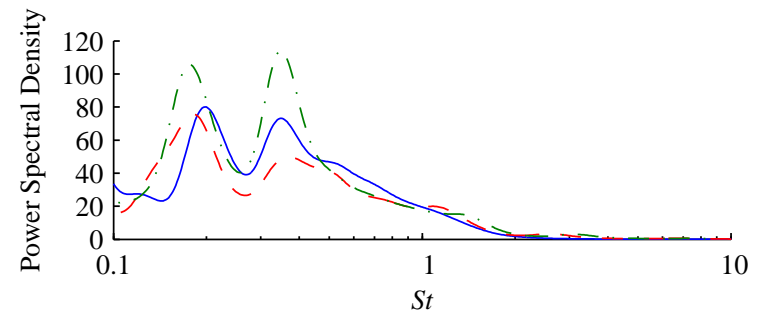

Figure 8. Wavelet spectra of short pressure time series (red, dashed), long pressure time series (green, dot-dash) and image pixel-intensity (blue, solid) for $\sigma=0.5$. The long pressure time series more accurately resolves the shedding spectra; however, the shorter series both identify the two shedding frequencies.
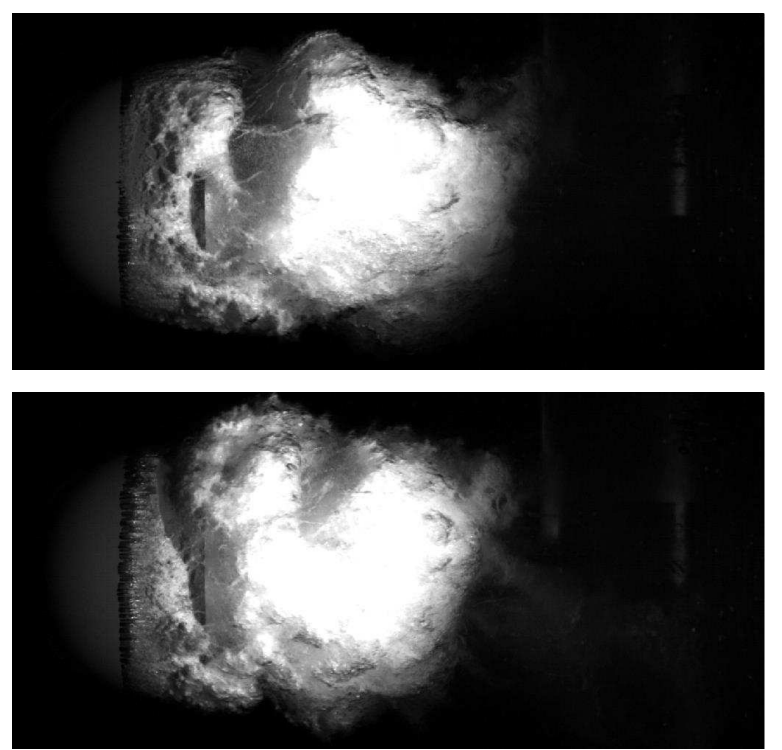

Figure 9. High-speed images of cavity shedding at $\sigma=0.5$. The top image shows a longer duration shedding event at $t=0.063 \mathrm{~s}$. The bottom image shows a shorter duration shedding event at $t=0.195 \mathrm{~s}$.

\section{Acknowledgements}

The authors wish to acknowledge the support of the University of Tasmania and the Defence Science and Technology Organisation. Wavelet software was provided by C. Torrence and G. Compo and is available at URL: http://paos.colorado.edu/research/wavelets/

\section{References}

[1] Arakeri, V. H. and Acosta, A. J., Viscous effects in the inception of cavitation on axisymmetric bodies, Journal of Fluids Engineering, 95, 1973, 519-527.

[2] Brandner, P. A., Walker, G. J., Niekamp, P. N. and Anderson, B., An investigation of cloud cavitation about a sphere, in 16th Australasian Fluid Mechanics Conference, Gold Coast, Australia, 2007, 1392-1398.

[3] Brandner, P. A., Walker, G. J., Niekamp, P. N. and Anderson, B., An experimental investigation of cloud cavitation about a sphere, Journal of Fluid Mechanics, 656, 2010, 147-176.

[4] Brennen, C. E., Cavity surface wave patters and general appearance, Journal of Fluid Mechanics, 44, 1970, 33 49.
[5] Brennen, C. E., Colonius, T., Wang, Y. C. and Preston, A., Cloud cavitation phenomena, in 22nd Symposium on Naval Hydrodynamics, Washington, D.C., 1998, 239-253.

[6] Ceccio, S. L. and Brennen, C. E., Dynamics of attached cavities on bodies of revolution, Journal of Fluids Engineering, 114, 1992, 93-99.

[7] de Lange, D. F., de Bruim, G. J. and van Wijngaarden, L., On the mechanism of cloud cavitation - experiment and modelling, in 2nd International Symposium on Cavitation, 1994, 45-49.

[8] Hofmann, M., Lohrber, H., Ludwig, G., Stoffel, B., Reboud, J. L. and Fortes-Patella, R., Numerical and experimental investigations on the self-oscillating behaviour of cloud cavitation. Part 1: Visualisation, in 3rd ASME/JSME Joint Fluids Engineering Conference, San Francisco, Calafornia, 1999.

[9] Huang, T. T., Cavitation observations on axisymmetric headforms, in ASME International Symposium on Cavitation Inception, New York, 1979, 51-61.

[10] Huang, T. T., Cavitation inception observations on six axisymmetric headforms, Journal of Fluids Engineering, 103, 1981, 273-279.

[11] Ito, Y., Seto, K. and Nagasaki, T., Periodical shedding of cloud cavitation from a single hydrofoil in high-speed cryogenic channel flow, Journal of Thermal Science, 18, 2009, 58-64.

[12] Kjeldsen, M., Arndt, R. E. A. and Effertz, M., Spectral characteristics of sheet/cloud cavitation, Journal of Fluids Engineering, 122, 2000, 481-487.

[13] Torrence, C. and Compo, G. P., A practical guide to wavelet analysis, Bulletin of the American Meteorological Society, 79, 1998, 61-78. 